\title{
Mismatch repair deficiency as a prognostic factor in mucinous colorectal cancer
}

\author{
Juliana Andrici ${ }^{1,2}$, Mahtab Farzin ${ }^{1,3}$, Loretta Sioson ${ }^{1,2}$, Adele Clarkson ${ }^{1,3}$, Nicole Watson ${ }^{1}$, \\ Christopher W Toon ${ }^{1,2,4}$ and Anthony J Gill ${ }^{1,2,3,5}$ \\ ${ }^{1}$ Cancer Diagnosis and Pathology Research Group, Kolling Institute of Medical Research, St Leonards, \\ NSW, Australia; ${ }^{2}$ Sydney Medical School, University of Sydney, Sydney, NSW, Australia; ${ }^{3}$ Department of \\ Anatomical Pathology, Royal North Shore Hospital, St Leonards, NSW, Australia; ${ }^{4}$ Histopath Pathology, \\ North Ryde, NSW, Australia and ${ }^{5}$ Sydney Vital Translational Research Centre, Royal North Shore Hospital, \\ St Leonards, NSW, Australia
}

\begin{abstract}
There is some uncertainty about pathological grading of mucinous colorectal adenocarcinoma, defined as colorectal cancer demonstrating at least $50 \%$ mucinous differentiation. Under the WHO 2000 classification mucinous colorectal cancer was considered high grade. However under the current WHO 2010 classification microsatellite unstable/mismatch repair-deficient (MSI/MMRd) mucinous colorectal cancer is considered low grade, whereas microsatellite stable/mismatch repair proficient (MSS/MMRp) tumours are high grade. However there is little empirical evidence for this approach. We therefore compared the long term survival of patients with MSI/MMRd vs MSS/MMRp mucinous colorectal cancer in a large unselected cohort of patients undergoing surgery at our institution from 1998 to 2011. There were 2608 patients in the cohort, of which 264 (10.1\%) were mucinous. $95(36 \%)$ of the mucinous tumours were microsatellite unstable. The all-cause 5-year survival of mucinous MSI/MMRd colorectal cancer was similar to that of non-mucinous low-grade colorectal cancer (73 vs $67 \%, P=0.368)$, and significantly better than that of both non-mucinous high-grade $(73$ vs $53 \%, P<0.001)$ and mucinous MSS/MMRp colorectal cancer (73 vs $57 \%, P=0.023$ ). The 5 -year survival of mucinous MSS/MMRp colorectal cancer was slightly better than that of non-mucinous high-grade patients (57 vs $53 \%, P=0.027)$, but significantly worse than that of non-mucinous low-grade colorectal cancer (57 vs $67 \%, P=0.018)$. In multivariate Cox regression analysis, conventional histological grade based on glandular differentiation maintained prognostic significance $(P=0.003)$ whereas MSI/MMRd status just failed to be statistically significant $(P=0.062)$. Our findings support the WHO 2010 approach that as a group mucinous MSS/MMRp colorectal cancers are biologically aggressive. However, grading based exclusively on MSI/MMR status may be overly simplistic as conventional grading based on the degree of glandular differentiation still holds greater prognostic significance in multivariate analysis.
\end{abstract}

Modern Pathology (2016) 29, 266-274; doi:10.1038/modpathol.2015.159; published online 15 January 2016

Mucinous colorectal adenocarcinoma is usually defined as colorectal cancer in which extracellular mucin comprises $>50 \%$ of the tumour volume. ${ }^{1}$ Mucinous colorectal cancer has been reported to account for $5-15 \%$ of colorectal carcinomas, ${ }^{2}$ and compared with non-mucinous colorectal cancer more commonly demonstrates microsatellite instability/DNA mismatch repair deficiency (MSI/ MMRd). ${ }^{3,4}$ In fact between 29 (ref. 5) and $42 \%{ }^{6}$ of mucinous colorectal cancers have been reported to demonstrate MSI/MMRd compared with $20 \%$ or less

Correspondence: Dr AJ Gill, MD, FRCPA, Department of Anatomical Pathology, Royal North Shore Hospital, Pacific Highway, St Leonards, NSW 2065, Australia. Email: affgill@med.usyd.edu.au Received 4 September 2015; revised 1 December 2015; accepted 2 December 2015; published online 15 January 2016 of all unselected colorectal cancers. ${ }^{7}$ Although some studies have reported worse survival outcomes and poorer response to chemotherapy in mucinous colorectal cancer, 8,9 to date findings have been inconsistent, with some studies showing no mortality association with mucinous histology. ${ }^{10-12}$

Pathological grading of mucinous CRC is problematic. Under conventional systems, colorectal cancer is graded based on the degree of glandular differentiation with cases showing $>50 \%$ gland formation being considered as low grade and cases showing $<50 \%$ glands being considered as high grade. ${ }^{1}$ However, historically it has been unclear whether this grading scheme should be applied to mucinous carcinomas. In fact, under the WHO 2000 classification all mucinous colorectal cancers were considered as high grade. ${ }^{13}$ However, under the 

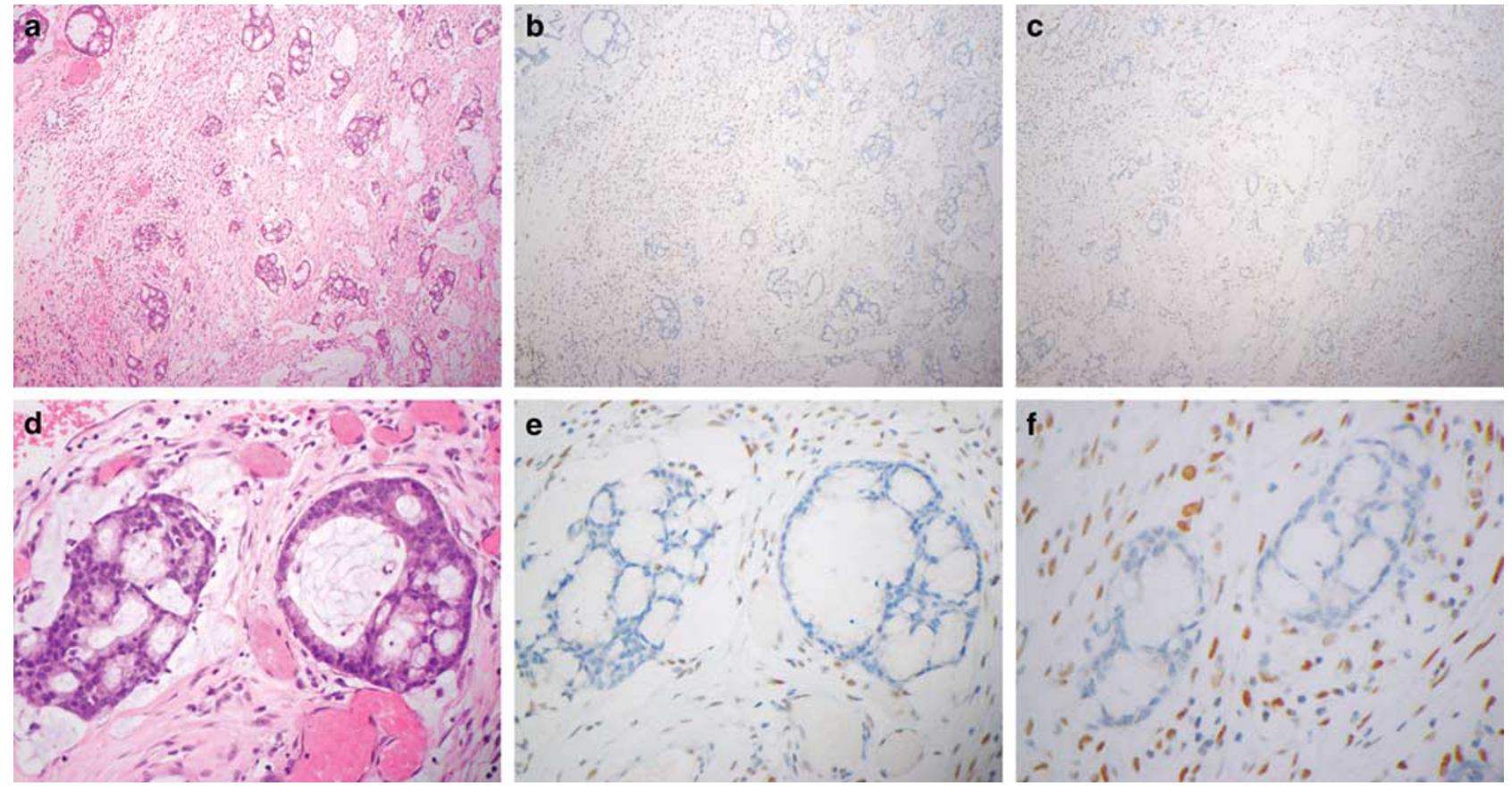

Figure 1 Serial H\&E (a and d), PMS2 (b and e) and MLH1 (c and f) stained sections of a mucinous MMRd colorectal adenocarcinoma. All the neoplastic cells show completely negative staining for both PMS2 and MLH1. Positive nuclear staining for both markers in non-neoplastic stromal and inflammatory cells is noted and act as internal positive controls (original magnifications: a-c $\times 100 ; \mathbf{d}-\mathbf{f} \times 400$ ).

WHO 2010 guidelines it is recommended that mucinous colorectal cancers should be graded on the basis of microsatellite instability/DNA mismatch repair status. ${ }^{1}$ That is, it is recommended that MSI/MMRd mucinous colorectal cancers should be considered as low grade, whereas microsatellite stable/mismatch repair proficient (MSS/MMRp) tumours should be considered as high grade regardless of cytoarchitectural features. However, while there is strong empirical evidence that MSI/MMRd colorectal cancers as a group have a significantly better prognosis than MSS/MMRp colorectal cancers as a group, ${ }^{14-16}$ the evidence that mucinous colorectal cancers can be graded based exclusively on microsatellite instability status is based on smaller studies or subgroup analysis and is less clear-cut. ${ }^{5,6,14-16}$

For example, based on only 60 mucinous colorectal cancers Leopoldo et $a l^{6}$ suggested that mucinous colorectal cancers had a better prognosis in a univariate model, but did not test this in a multivariate model. Similarly, Messerini et al ${ }^{17}$ studied 50 mucinous CRCs and found that microsatellite unstable mucinous colorectal cancer had a better prognosis in univariate analysis; however, microsatellite instability held no prognostic significance in mucinous colorectal cancer in a multivariate model predominantly because of a strong association with a less aggressive (pushing) growth pattern and lower stage. In fact only very recently and based on only 84 tumours, Yoon et $a l^{5}$ were able to demonstrate a lower 4-year recurrence and better overall survival rates for mucinous microsatellite unstable colorectal cancer vs mucinous microsatellite stable colorectal cancer $(P=0.018$ and $P=0.046)$.

In view of the limited evidence in this area, we therefore sought to investigate whether mucinous colorectal cancers should be graded on the basis of microsatellite instability/mismatch repair status by comparing the outcomes of mucinous MSI/MMRd, mucinous MSS/MMRp, and conventional non-mucinous low- and high-grade colorectal cancer in a large unselected cohort of patients undergoing surgery with curative intent.

\section{Materials and methods}

The computerized database of the department of Anatomical Pathology Royal North Shore Hospital, Sydney, Australia was searched for all cases of resected colorectal cancer between 1998 and 2011. This cohort has previously been described. ${ }^{18}$ Briefly, we included all consecutive cases of colorectal cancer treated surgically with curative intent. Endoluminally-resected tumours and histologies other than adenocarcinoma and its variants as defined by $\mathrm{WHO}^{1}$ were excluded. In accordance with the WHO 2010 classification, mucinous histology was defined as extracellular mucin comprising $>50 \%$ of the tumour. ${ }^{1}$ Tumours that demonstrated no or $<50 \%$ mucinous differentiation were considered as non-mucinous. In view of the colon continuum model advanced by Yamauchi et al, ${ }^{19}$ tumour location was divided into six groups: caecum, ascending colon, transverse colon, descending colon, sigmoid colon, 

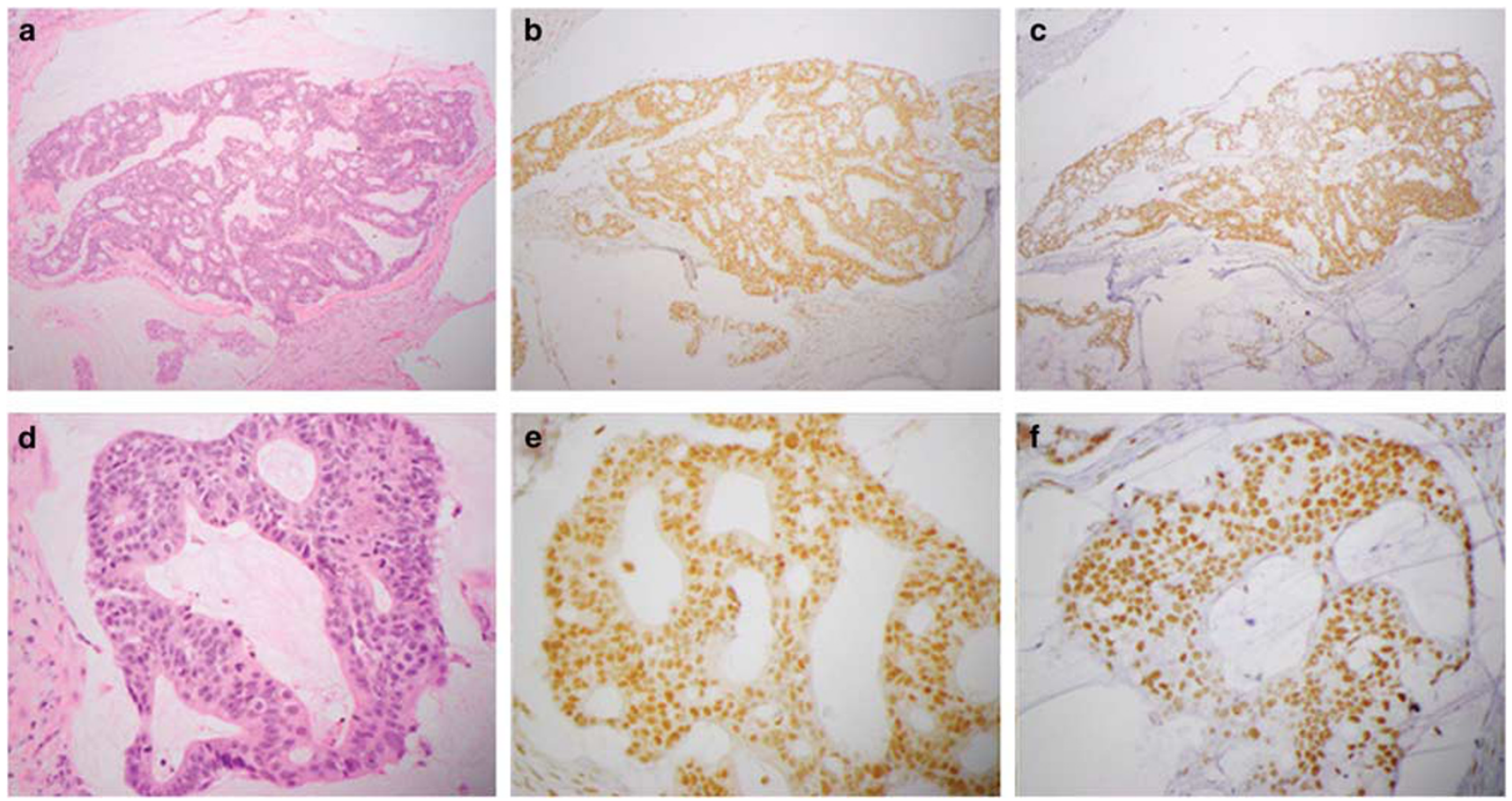

Figure 2 Serial H\&E (a and d), PMS2 (b and e), and MLH1 (c and f) stained sections of a mucinous MMRp colorectal adenocarcinoma. In this case, the neoplastic cells demonstrate preserved positive staining for PMS2 and MLH1. This tumour also demonstrated positive staining for the other mismatch repair markers (MSH2 and MSH6) (original magnifications: $\mathbf{a}-\mathbf{c} \times 100 ; \mathbf{d}-\mathbf{f} \times 400$ ).

and rectum. All tumours were restaged according to the 7 th edition 2010 AJCC staging system. ${ }^{20}$ Tumours underwent conventional histological grading based on the degree of glandular differentiation, with tumours demonstrating $<50 \%$ gland formation being considered as high grade.

A tissue microarray containing two $1 \mathrm{~mm}$ cores of formalin-fixed paraffin-embedded tissue from each tumour was created, and immunohistochemistry for four mismatch repair markers (PMS2, MLH1, MSH2, and MSH6) was performed on the tissue microarray sections using previously described methods. ${ }^{7,21}$ Negative staining (MMRd/MSI) was defined as completely absent nuclear staining in the neoplastic cells in the presence of a positive internal control (Figure 1), while the MMRp/MSS pattern was defined by positive nuclear staining for all four markers in neoplastic cells (Figure 2). In our hands, we have previously demonstrated excellent concordance between immunohistochemistry interpreted on tissue microarrays and whole sections, ${ }^{18}$ and if there was uncertainty about interpretation of the immunohistochemistry, stains were repeated on whole sections. If all four mismatch repair markers demonstrated positive staining, then the tumour was considered as MMRp/MSS. If one of the markers demonstrated negative staining in the presence of a positive internal control, then the tumour was considered as MMRd/MSI. We note that we tested for mismatch repair status by immunohistochemistry, rather than for microsatellite instability by molecular means. Mutation-specific immunohistochemistry for BRAFV600E was also performed with a mutation-specific mouse monoclonal antibody (clone VE1, SpringBioscience, Pleasonton, CA, USA) at a dilution of 1 in 80 using identical methods to that which we have previously described. ${ }^{18}$ Briefly, immunohistochemistry was performed using the Leica BondIII autostainer (Leica Microsystems, Mount Waverley, VIC, Australia) with alkaline antigen retrieval (solution ER2, VBS part no: AR9640, Leica Microsystems). We have previously demonstrated that, in our hands, BRAFV600E mutationspecific immunohistochemistry is highly concordant with formal molecular testing when performed on both whole and tissue microarray sections. ${ }^{18}$

Survival data were obtained from hospital medical records, surgeons' private rooms, and publicly available death notices. Overall survival was defined as the time from the date of surgical resection for the initial colorectal cancer presentation until the date of last contact or death. Clinical and pathological characteristics were compared using a Chi-Square test to compare differences in the frequency distributions of categorical study variables, and an independent samples $t$-test or one-way ANOVA to compare means of continuous variables such as age and tumour size between the different study groups. All-cause 5-year survival for the mucinous carcinoma cohort was analysed using the Kaplan-Meier method. Multivariate Cox regression was used to calculate the hazard ratio for death from any cause in a model which included age at diagnosis, gender, site, grade, stage, tumour size, BRAFV600E mutation, and mismatch repair status. Statistical analyses were performed using IBM SPSS Statistics v22. 
Table 1 Clinical and pathological characteristics of patients with mucinous colorectal cancer, and high- and low-grade non-mucinous colorectal cancer

\begin{tabular}{|c|c|c|c|c|c|}
\hline Characteristic & $\begin{array}{l}\text { Mucinous } \\
\text { tumours }\end{array}$ & $\begin{array}{l}\text { High-grade (non- } \\
\text { mucinous) tumours }\end{array}$ & $\begin{array}{c}\mathrm{P}-\text { value (mucinous vs } \\
\text { high grade) }\end{array}$ & $\begin{array}{l}\text { Low-grade (non- } \\
\text { mucinous) tumours }\end{array}$ & $\begin{array}{c}\text { P-value (mucinous vs } \\
\text { low grade) }\end{array}$ \\
\hline Age, mean (years) & 74.1 & 71.1 & 0.010 & 70.5 & $<0.001$ \\
\hline Female gender, $n(\%)$ & $157(59.5)$ & $253(54.3)$ & 0.176 & $902(48.0)$ & $<0.001$ \\
\hline MSI status & & & 0.001 & & $<0.001$ \\
\hline MSI & $95(36.0)$ & $147(31.5)$ & - & $182(9.7)$ & - \\
\hline MSS & $169(64.0)$ & $294(63.1)$ & - & $1559(83.0)$ & - \\
\hline Stage & & & 0.001 & & 0.001 \\
\hline I & $29(11.1)$ & $39(8.4)$ & - & $409(21.8)$ & - \\
\hline II & $102(38.8)$ & $112(26.2)$ & - & 675 (35.9) & - \\
\hline III & $119(45.2)$ & $263(56.4)$ & - & $730(38.9)$ & - \\
\hline IV & $13(4.9)$ & $42(9.0)$ & - & $61(3.2)$ & - \\
\hline Site & & & $<0.001$ & & $<0.001$ \\
\hline Caecum & $80(30.5)$ & $209(45.0)$ & - & $556(29.7)$ & - \\
\hline Ascending colon & $57(21.8)$ & $10(2.2)$ & - & $27(1.4)$ & - \\
\hline Transverse colon & $35(13.4)$ & $70(15.1)$ & - & $177(9.4)$ & - \\
\hline Descending colon & $8(3.1)$ & $22(4.7)$ & - & $103(5.5)$ & - \\
\hline Sigmoid colon & $34(13.0)$ & $64(13.8)$ & - & $485(25.9)$ & - \\
\hline Rectum & $48(18.3)$ & $89(19.2)$ & - & $527(28.1)$ & - \\
\hline Lymph-node & & & $<0.001$ & & 0.056 \\
\hline metastasis & & & & & \\
\hline Yes & $119(45.1)$ & $290(62.2)$ & - & 731 (38.9) & - \\
\hline No & $145(54.9)$ & $176(37.8)$ & - & $1147(61.1)$ & - \\
\hline$B R A F$ status & & & 0.018 & & $<0.001$ \\
\hline BRAF negative & $171(64.8)$ & $248(55.7)$ & - & $1512(86.2)$ & - \\
\hline BRAF positive & $93(35.2)$ & $197(44.3)$ & - & $242(13.8)$ & - \\
\hline Tumour size (mm) & 50.8 & 48.9 & 0.304 & 40.5 & $<0.001$ \\
\hline
\end{tabular}

A $P$-value of $<0.05$ was considered as statistically significant. This study was approved by the Northern Sydney Local Health District medical ethics review board.

\section{Results}

We identified 2608 patients with colorectal cancer during the period between 1998 and 2011. In all, 264 $(10.1 \%)$ of cases were mucinous adenocarcinomas. The clinical and pathological features are presented in Table 1. Briefly, mucinous tumours were more similar to high-grade non-mucinous colorectal cancer than to low-grade tumours in their association with older age, female gender, site of occurrence in the more proximal colon (caecum and ascending colon), and larger tumour size. When analysing all-cause 5-year survival, the survival rate of the entire mucinous colorectal cancer cohort was intermediate between low-grade and high-grade non-mucinous carcinoma, but much more closely approximated that of low-grade non-mucinous tumours (63 vs $67 \%$, $P=0.181$ ). The overall survival of mucinous colorectal cancer as a group was statistically significantly

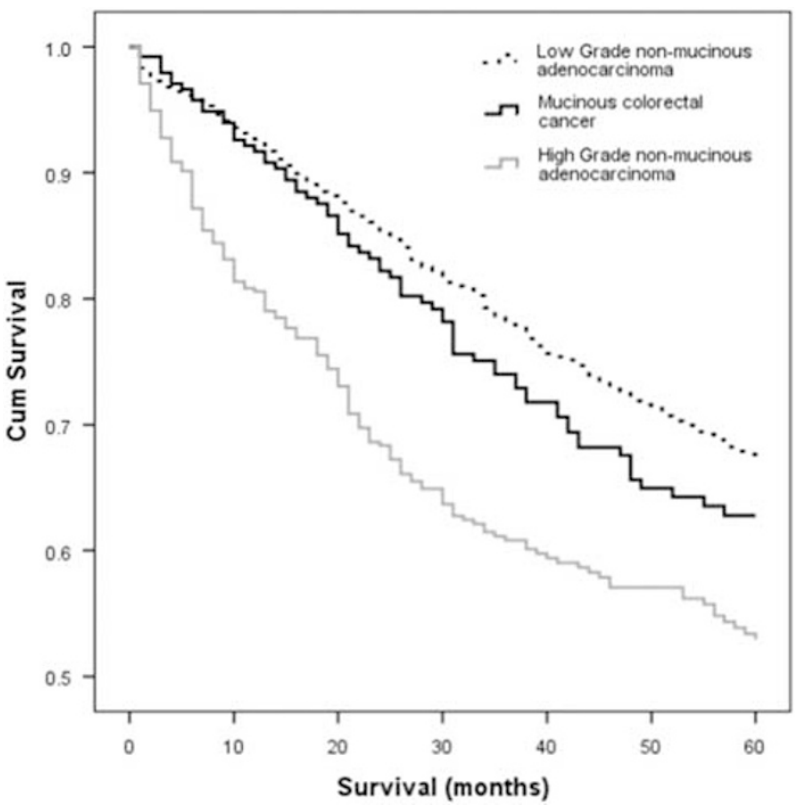

Figure 3 Five-year Kaplan-Meier survival curves for mucinous colorectal cancer, high-grade non-mucinous colorectal cancer, and low-grade non-mucinous colorectal cancer. As a group, the survival of mucinous carcinomas is intermediate between lowgrade and high-grade non-mucinous carcinomas. 
Table 2 Clinical and pathological characteristics of patients with mucinous MSI and high- and low-grade non-mucinous CRC

\begin{tabular}{|c|c|c|c|c|c|}
\hline Characteristic & Mucinous MSI & $\begin{array}{c}\text { High-grade } \\
\text { (non-mucinous) tumours }\end{array}$ & $\begin{array}{c}\text { P-value } \\
\text { (MSI vs high grade) }\end{array}$ & $\begin{array}{c}\text { Low-grade } \\
\text { (non-mucinous) tumours }\end{array}$ & $\begin{array}{c}\text { P-value } \\
\text { (MSI vs low grade) }\end{array}$ \\
\hline Age, mean (years) & 77.0 & 71.7 & $<0.001$ & 70.5 & $<0.001$ \\
\hline Female gender, $n(\%)$ & $68(71.6)$ & $253(54.3)$ & 0.002 & $902(48.0)$ & $<0.001$ \\
\hline Stage & & & $<0.001$ & & 0.100 \\
\hline I & $14(14.7)$ & $39(8.4)$ & - & 409 (21.8) & - \\
\hline II & 47 (49.5) & $112(26.2)$ & - & $675(35.9)$ & - \\
\hline III & $32(33.7)$ & $263(56.4)$ & - & $730(38.9)$ & - \\
\hline IV & $2(2.1)$ & $42(9.0)$ & - & $61(3.2)$ & - \\
\hline Site & & & $<0.001$ & & $<0.001$ \\
\hline Caecum & $39(41.5)$ & $209(45.0)$ & - & $556(29.7)$ & - \\
\hline Ascending colon & $24(25.5)$ & $10(2.2)$ & - & $27(1.4)$ & - \\
\hline Transverse colon & $22(23.4)$ & $70(15.1)$ & - & $177(9.4)$ & - \\
\hline Descending colon & $0(0)$ & $22(4.7)$ & - & $103(5.5)$ & - \\
\hline Sigmoid colon & $6(6.4)$ & $64(13.8)$ & - & $485(25.9)$ & - \\
\hline Rectum & $3(3.2)$ & $89(19.2)$ & - & $527(28.1)$ & - \\
\hline Lymph-node metastasis & & & $<0.001$ & & 0.151 \\
\hline Yes & $30(31.6)$ & $290(62.2)$ & - & 731 (38.9) & - \\
\hline No & $65(68.4)$ & $176(37.8)$ & - & $1147(61.1)$ & - \\
\hline BRAF status & & & $<0.001$ & & $<0.001$ \\
\hline BRAF negative & $34(35.8)$ & $248(55.7)$ & - & $1512(86.2)$ & - \\
\hline BRAF positive & $61(64.2)$ & $197(44.3)$ & - & $242(13.8)$ & - \\
\hline Tumour size, mean (mm) & 52.9 & 48.9 & 0.147 & 40.5 & $<0.001$ \\
\hline
\end{tabular}

better than that of high-grade non-mucinous tumours (63 vs 53\%, $P<0.001$ ) (Figure 3).

Of the 2068 colorectal cancers, 1024 (49.5\%) had mismatch repair immunohistochemistry performed on whole sections at the time of primary resection. In all, 166 (63\%) of the 264 patients in the mucinous cohort had immunohistochemistry performed on whole sections at the time of primary diagnosis. In a total of $50(2.4 \%)$ of 2068 cases, immunohistochemistry was uninterpretable on tissue microarray sections for the reasons stated above and had not been performed as part of routine clinical care and therefore had to be repeated on whole sections for this study.

MMRd was present in $36.0 \%$ of mucinous colorectal cancer, compared with only $14.1 \%$ on non-mucinous tumours. The clinical and pathological characteristics of mucinous MMRp and MMRd tumours are presented in Tables 2 and 3. Briefly, patients with mucinous MMRd colorectal cancers were older, were more likely to be female, and the tumours arose more often in the more proximal colon (caecum and ascending colon), compared to patients with either low- or high-grade non-mucinous tumours. Patients with mucinous MMRp tumours were similar to patients with non-mucinous high-grade tumours in terms of age, gender distribution, and BRAF mutation status. The all-cause 5-year survival of mucinous MMRd patients was similar to that of non-mucinous low-grade cancer patients (73 vs $67 \%, P=0.367$ ), and statistically significantly better than that of both non-mucinous high-grade (73 vs 53\%, $P<0.001$ ) and mucinous MMRp patients (73 vs 57\%, $P=0.023$ ).
(Figure 4) Although the clinical and pathological characteristics of mucinous MMRp tumours appeared more similar to high-grade non-mucinous tumours (Tables 3 and 4), the 5-year survival of mucinous MMRp patients was better than that of non-mucinous high-grade patients (57 vs $53 \%, P=0.027$ ). However, the 5-year survival of mucinous MMRp patients was still statistically significantly lower than that of non-mucinous low-grade patients (57 vs 67\%, $P=0.018$ ). On sub-grouping of the MMRp tumours by BRAF status, we found no statistically significant difference in 5-year survival between BRAF-negative MMRp colorectal cancers (57\%) and BRAF-positive MMRp (51\%) tumours $(P=0.610)$.

When analysing the clinical and pathologic characteristics of the patient cohort by tumour location (Table 5), we found that patients with more distal tumours (sigmoid colon and rectum) trended to be younger males. Additionally, the tumours found in these more distal locations tended to be smaller and BRAF negative. The more distal mucinous tumours were also much more likely to be microsatellite stable/MMRp. Data on the number of mucinous mismatch repair-deficient tumours associated with Lynch syndrome were not available.

Multivariate Cox regression analysis assessing the risk of death from any cause in a model including age, gender, site, stage, traditional tumour grade, microsatellite instability/mismatch repair status, BRAF mutation status, and size is presented in Table 6 . Briefly in multivariate analysis, older age (HR 1.04; 95\% CI, 1.01-1.07, $P=0.006$ ), stage IV tumours (HR 10.50; $95 \%$ CI, 1.84-59.83, $P=0.008$ ), and traditional 
Table 3 Clinical and pathological characteristics of patients with mucinous MSS and high- and low-grade non-mucinous CRC

\begin{tabular}{|c|c|c|c|c|c|}
\hline Characteristic & $\begin{array}{l}\text { Mucinous } \\
\quad \text { MSS }\end{array}$ & $\begin{array}{l}\text { High-grade (non- } \\
\text { mucinous) tumours }\end{array}$ & $\begin{array}{c}\text { P-value (mucinous vs } \\
\text { high-grade) }\end{array}$ & $\begin{array}{l}\text { Low-grade (non- } \\
\text { mucinous) tumours }\end{array}$ & $\begin{array}{c}\text { P-value (mucinous vs } \\
\text { low-grade) }\end{array}$ \\
\hline Age, mean (years) & 72.5 & 71.7 & 0.465 & 70.5 & 0.045 \\
\hline Female gender, $n(\%)$ & $89(52.7)$ & $253(54.3)$ & 0.716 & $902(48.0)$ & 0.248 \\
\hline Stage & & & 0.344 & & $<0.001$ \\
\hline I & $15(8.9)$ & $39(8.4)$ & - & $409(21.8)$ & - \\
\hline II & $55(32.7)$ & $112(26.2)$ & - & $675(35.9)$ & - \\
\hline III & $87(51.8)$ & $263(56.4)$ & - & $730(38.9)$ & - \\
\hline IV & $11(6.5)$ & $42(9.0)$ & - & $61(3.2)$ & - \\
\hline Site & & & $<0.001$ & & $<0.001$ \\
\hline Caecum & $41(24.4)$ & $209(45.0)$ & - & $556(29.7)$ & - \\
\hline Ascending colon & 33 (19.6) & $10(2.2)$ & - & $27(1.4)$ & - \\
\hline Transverse colon & $13(7.7)$ & $70(15.1)$ & - & $177(9.4)$ & - \\
\hline Descending colon & $8(4.8)$ & $22(4.7)$ & - & $103(5.5)$ & - \\
\hline Sigmoid colon & $28(16.7)$ & $64(13.8)$ & - & $485(25.9)$ & - \\
\hline Rectum & $45(26.8)$ & $89(19.2)$ & - & $527(28.1)$ & - \\
\hline Lymph-node metastasis & & & 0.030 & & $<0.001$ \\
\hline Yes & $89(52.7)$ & $290(62.2)$ & - & $731(38.9)$ & - \\
\hline No & $80(47.3)$ & $176(37.8)$ & - & $1147(61.1)$ & - \\
\hline BRAF status & & & $<0.001$ & & 0.047 \\
\hline BRAF negative & $137(81.1)$ & $248(55.7)$ & - & $1512(86.2)$ & - \\
\hline BRAF positive & $32(18.9)$ & $197(44.3)$ & - & $242(13.8)$ & - \\
\hline Tumour size, mean (mm) & 49.6 & 48.9 & 0.777 & 40.5 & $<0.001$ \\
\hline
\end{tabular}

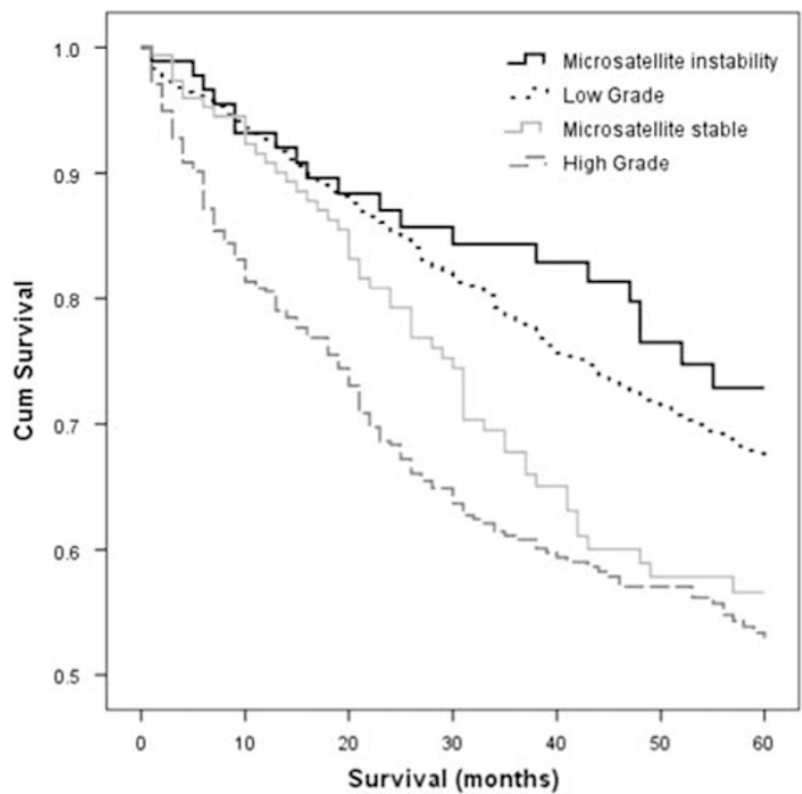

Figure 4 Five-year Kaplan-Meier survival curves for MMRd mucinous colorectal cancer, MMRp mucinous colorectal cancer, high-grade non-mucinous colorectal cancer, and low-grade non-mucinous colorectal cancer. The survival of mucinous MMRd colorectal carcinoma is slightly better than non-mucinous low-grade carcinoma, whereas the survival of mucinous MMRp colorectal carcinoma is more similar to non-mucinous high-grade carcinoma.

histological high grade (HR 2.66; 95\% CI, 1.38-5.12, $P=0.003$ ) were associated with worse survival outcomes. In this multivariate model, the survival advantage for mucinous MMRd tumours just failed to
Table 4 Clinical and pathological characteristics of patients with mucinous MSI and MSS colorectal tumours

\begin{tabular}{lccr}
\hline Characteristic & Mucinous MSI & Mucinous MSS & P-value \\
\hline$N(\%)$ & $95(36.0)$ & $169(64.0)$ & \\
Age, mean (range) & 77.0 & 72.5 & 0.004 \\
Female gender, $n$ (\%) & $68(71.6)$ & $89(52.7)$ & 0.003 \\
Stage & & & \\
I & $14(14.7)$ & $15(8.9)$ & \\
II & $47(49.5)$ & $55(32.7)$ & \\
III & $32(33.7)$ & $87(51.8)$ & \\
IV & $2(2.1)$ & $11(6.5)$ & \\
& & & \\
Site & & & \\
Caecum & $39(41.5)$ & $41(24.4)$ & \\
Ascending colon & $24(25.5)$ & $33(19.6)$ & \\
Transverse colon & $22(23.4)$ & $13(7.7)$ & \\
Descending colon & $0(0)$ & $8(4.8)$ & \\
Sigmoid colon & $6(6.4)$ & $28(16.7)$ & \\
Rectum & $3(3.2)$ & $45(26.8)$ & \\
BRAF status & & & \\
BRAF negative & $34(35.8)$ & $137(81.1)$ & \\
BRAF positive & $61(64.2)$ & $32(18.9)$ & \\
Tumour size, mean (mm) & 52.9 & 49.6 & 0.001 \\
& & & \\
\hline
\end{tabular}

reach statistical significance (HR $0.49 ; 95 \%$ CI, $0.23-1.04, P=0.062$ ).

\section{Discussion}

In this study, we provide strong empirical support for the WHO 2010 premise that microsatellite instability/mismatch repair status is a powerful 
Table 5 Clinical and pathological characteristics of colorectal cancer patients according to tumour location ( $n=2608$ )

\begin{tabular}{|c|c|c|c|c|c|c|c|}
\hline Characteristic & Caecum & Ascending colon & Transverse colon & Descending colon & Sigmoid colon & Rectum & $\mathrm{P}$-value \\
\hline$N(\%)$ & $845(32.4)$ & $94(3.6)$ & $282(10.8)$ & $133(5.1)$ & $583(22.4)$ & $664(25.5)$ & \\
\hline Age, mean (years) & 74.1 & 74.4 & 73.0 & 71.4 & 69.7 & 67.0 & $<0.001$ \\
\hline Gender, n (\%) & & & & & & & $<0.001$ \\
\hline Male & 367 (43.4) & $34(36.2)$ & $124(44.0)$ & $65(48.9)$ & $315(54.0)$ & $387(58.3)$ & \\
\hline Female & $478(56.6)$ & $60(63.8)$ & $158(56.0)$ & $68(51.1)$ & $268(46.0)$ & $277(41.7)$ & \\
\hline Histology & & & & & & & $<0.001$ \\
\hline Mucinous & $80(9.5)$ & $57(60.6)$ & $35(12.4)$ & $8(6.0)$ & $34(5.8)$ & $48(7.2)$ & \\
\hline Non-mucinous & $765(90.5)$ & $37(39.4)$ & $247(87.6)$ & $125(94.0)$ & $549(94.2)$ & $616(92.8)$ & \\
\hline adenocarcinoma & & & & & & & \\
\hline $\begin{array}{l}\text { MSI status (for mucinous } \\
\text { tumours) }\end{array}$ & & & & & & & $<0.001$ \\
\hline MSI & $235(29.2)$ & $40(43.5)$ & $89(33.0)$ & $14(11.2)$ & $32(5.8)$ & $12(2.0)$ & \\
\hline MSS & $570(70.8)$ & $52(56.5)$ & $181(67.0)$ & $111(88.8)$ & $520(94.2)$ & $584(98.0)$ & \\
\hline BRAF status & & & & & & & $<0.001$ \\
\hline BRAF negative & $550(68.0)$ & $52(55.9)$ & $157(57.5)$ & $103(83.1)$ & 495 (89.5) & $570(94.2)$ & \\
\hline BRAF positive & $259(32.0)$ & $41(44.1)$ & $116(42.5)$ & $21(16.9)$ & $58(10.5)$ & $35(5.8)$ & \\
\hline Stage & & & & & & & $<0.001$ \\
\hline I & $134(15.9)$ & $10(10.6)$ & $32(11.3)$ & $26(19.5)$ & $96(16.5)$ & $177(26.7)$ & \\
\hline II & $310(36.7)$ & $45(47.9)$ & $118(41.8)$ & $46(34.6)$ & $204(35.0)$ & $174(26.2)$ & \\
\hline III & $347(37.2)$ & $35(37.2)$ & $114(40.4)$ & $55(41.4)$ & $262(44.9)$ & $297(44.8)$ & \\
\hline IV & $52(6.2)$ & $4(4.3)$ & $18(6.4)$ & $6(4.5)$ & $21(3.6)$ & $15(2.3)$ & \\
\hline Tumour size (mm) & 46.8 & 50.3 & 47.0 & 44.7 & 41.1 & 37.33 & $<0.001$ \\
\hline
\end{tabular}

Table 6 Univariate and multivariate Cox regression proportional hazards analysis for all-cause mortality of 264 mucinous carcinoma patients

\begin{tabular}{|c|c|c|}
\hline Variable & $\begin{array}{c}\text { Univariate cox regression } \\
\text { HR }(95 \% \text { CI), P-value }\end{array}$ & $\begin{array}{c}\text { Multivariate cox regression } \\
\text { HR }(95 \% \text { CI), P-value }\end{array}$ \\
\hline Age at diagnosis & 1.03 (1.01-1.05), 0.004 & 1.04 (1.01-1.07), 0.006 \\
\hline \multicolumn{3}{|l|}{ Gender } \\
\hline Male & 1.00 & 1.00 \\
\hline Female & $0.80(0.52-1.24), 0.316$ & $0.80(0.44-1.43), 0.449$ \\
\hline \multicolumn{3}{|l|}{ Site } \\
\hline Caecum & 1.00 & 1.00 \\
\hline Ascending colon & $0.71(0.37-1.35), 0.298$ & $1.16(0.53-2.58), 0.704$ \\
\hline Transverse colon & $1.04(0.50-2.14), 0.925$ & $1.81(0.74-4.45), 0.196$ \\
\hline Descending colon & 1.38 (0.48-3.95), 0.551 & $0.22(0.04-1.32), 0.098$ \\
\hline Sigmoid colon & $1.02(0.50-2.05), 0.996$ & $0.94(0.32-2.74), 0.911$ \\
\hline Rectum & $0.94(0.52-1.72), 0.849$ & $1.13(0.49-2.60), 0.772$ \\
\hline \multicolumn{3}{|l|}{ Overall stage } \\
\hline Stage I & 1.00 & 1.00 \\
\hline Stage II & $1.68(0.68-4.15), 0.258$ & 1.06 (0.30-3.83), 0.926 \\
\hline Stage III & $2.55(1.07-6.06), 0.035$ & $2.07(0.57-7.48), 0.270$ \\
\hline Stage IV & $10.80(3.72-31.29),<0.001$ & $10.50(1.84-59.83), 0.008$ \\
\hline \multicolumn{3}{|l|}{ Tumour grade } \\
\hline Low grade & 1.00 & 1.00 \\
\hline High grade & 2.18 (1.22-3.89), 0.008 & 2.66 (1.38-5.12), 0.003 \\
\hline \multicolumn{3}{|l|}{ MSI status } \\
\hline MSS & 1.00 & 1.00 \\
\hline MSI & 0.66 (0.41-1.04), 0.071 & $0.49(0.23-1.04), 0.062$ \\
\hline \multicolumn{3}{|l|}{ BRAF status } \\
\hline BRAF negative & 1.00 & 1.00 \\
\hline BRAF positive & $0.80(0.51-1.26), 0.341$ & $1.18(0.56-2.46), 0.666$ \\
\hline Tumour size & 1.004 (0.994-1.013), 0.431 & 0.998 (0.985-1.012), 0.828 \\
\hline
\end{tabular}


prognostic factor in mucinous tumours; however, it is clear that traditional cytoarchitectural grade is still prognostically important. The all-cause survival of mucinous MMRd colorectal cancers was similar to non-mucinous low-grade cancers, and in fact the mucinous MMRd patients demonstrated a slightly better 5-year survival, although this was not statistically significant $(P=0.181)$. In contrast, MMRp mucinous colorectal cancers demonstrated worse survival compared with both mucinous MMRd tumours $(P=0.023)$ and non-mucinous low-grade tumours $(P=0.018)$. Although the marginal benefit in 5-year survival of mucinous MMRp colorectal cancers compared with high-grade non-mucinous tumours reached statistical significance $(P=0.026)$, the difference in survival (57 vs $53 \%$ ) was appreciably smaller when compared with low-grade non-mucinous tumours $(67 \%)$ and mucinous MSI tumours $(73 \%)$. Put simply, and as summarized in Figure 4, the overall survival curve of mucinous MMRd colorectal cancer was similar to that of non-mucinous low-grade carcinomas, whereas the survival curves of mucinous MMRp colorectal cancer were similar to that of high-grade non-mucinous colorectal cancers. That is, our data support the approach that mucinous MMRd/MSI colorectal cancers behave similarly to low-grade non-mucinous colorectal cancers and mucinous MMRp/MSS colorectal cancers behave similarly to high-grade non-mucinous colorectal cancers.

However, we note that in multivariate analysis, conventional histological high grade was still associated with worse overall survival (HR 2.66; 95\% CI, 1.38-5.12, $P=0.003$ ) whereas the worse survival associated with MSS/MMRp status just failed to reach statistical significance $(0=0.062)$ when grade was included in the model. Therefore, we believe that there is still a role for conventional grading in mucinous colorectal carcinomas in addition to risk stratification on the basis of MSI/MMR status. That is, it is probably simplistic to grade mucinous colorectal cancers exclusively on the basis of microsatellite instability/mismatch repair status while ignoring cytoarchitectural features which are still highly prognostically significant.

In our study $36 \%$ of mucinous tumours demonstrated MMRd, which is in keeping with previous studies reporting ranges from 29 to $42 \%^{5,6}$ and suggests that our cohort is representative of mucinous colorectal cancers. Given that there is increasing evidence the BRAFV600E mutant microsatellite stable tumours are a particularly poor prognostic group, ${ }^{22-28}$ we attempted to further stratify mucinous MMRp tumours on the basis of BRAF status. Interestingly, and perhaps unexpectedly, we found similar 5-year survival rates for BRAF wild type $(57 \%)$ and BRAFV600E mutant (51\%) MMRp mucinous colorectal cancers $(P=0.610)$. That is, our findings suggest that mucinous MMRp tumours are aggressive regardless of BRAF status.
Both Yamauchi et $a 1^{19}$ and Ito et $a l^{29}$ assessed molecular and pathological characteristics of colorectal tumours at a continuum of sites along the colon rather than dividing the colon into right and left sides. A similar approach was employed in this study when categorizing tumour location, and our findings support the concept of a gradual evolution in molecular and pathologic characteristics along bowel sites, as opposed to a dichotomy created by a two-colon classification which divides the bowel into proximal and distal colon. In fact in terms of both $B R A F$ mutation and MSI status, our results are very similar to those of Yamauchi et al. ${ }^{19}$ In our study, MMRd- and BRAFV600E-mutated colorectal cancers were very infrequent in the rectum ( 2 and $5.8 \%$, respectively), increased slightly in the sigmoid (5.8 and 10.5\%), continued to increase in the descending colon (11.2 and $16.9 \%$ ) and transverse colon (33 and $42.5 \%$ ); peaked in the ascending colon (43.5 and $44.1 \%$ ) and then decreased in the caecum (29.2 and $32 \%)$. That is, although it was not the emphasis of our study, we note that our mismatch repair and BRAF findings also support the colorectal continuum model. ${ }^{19,29}$

As our study was designed to address the WHO 2010 premise that mucinous colorectal cancers can be graded on the basis of mismatch repair/microsatellite instability status, we used the WHO 2010 criteria for the diagnosis of mucinous carcinoma which requires that $>50 \%$ of the tumour demonstrate mucinous differentiation. ${ }^{1}$ However, we accept that tumours with $<50 \%$ mucinous differentiation may still demonstrate some or all of the characteristics of mucinous colorectal cancers and that further studies will be required to determine whether tumour with $<50 \%$ mucinous differentiation can be graded on the basis of microsatellite instability/ mismatch repair status.

In conclusion, our study supports the WHO 2010 premise that mucinous MMRd colorectal cancers have an outcome similar to low-grade non-mucinous tumours whereas mucinous MMRp colorectal cancers behave similarly to high-grade tumours. Although it is reasonable to consider mucinous MMRd/MSI colorectal cancers as biologically low grade and MMRp/ MSS mucinous colorectal cancers as biologically high grade, we note that conventional cytoarchitectural grade based on the degree of glandular differentiation is still a powerful prognostic marker in mucinous colorectal cancers independent of MSI/MMR status and should not be discarded.

\section{Acknowledgments}

This project was supported by the Sydney Vital, Translational Cancer Research, through a Cancer Institute NSW competitive grant.

\section{Disclosure/conflict of interest}

The authors declare no conflict of interest. 


\section{References}

1 Hamilton SR, Bosman FT, Boffetta P et al. Carcinoma of the colon and rectumBosman FT, Carneiro F, Hruban $\mathrm{RH}$, Theise ND et al. WHO Classification of Tumours of the Digestive System. 4th (edn). Lyon: IARC Press. 134-146.

2 Nozoe T, Anai H, Nasu S et al. Clinicopathological characteristics of mucinous carcinoma of the colon and rectum. J Surg Oncol 2000;75:103-107.

3 Chiang J-M, Chen M-C, Changchien C et al. Favorable influence of age on tumor characteristics of sporadic colorectal adenocarcinoma. Dis Colon Rectum 2003;46: 904-910.

4 Chiang J-M, Yeh C-Y, Changchien CR et al. Mucinous adenocarcinoma showing different clinicopathological and molecular characteristics in relation to different colorectal cancer subgroups. Int J Colorectal Dis 2010;25:941-947.

5 Yoon YS, Kim J, Hong SM et al. Clinical implications of mucinous components correlated with microsatellite instability in patients with colorectal cancer. Colorectal Dis 2015;17:0161-0167.

6 Leopoldo S, Lorena B, Cinzia et al. Two subtypes of mucinous adenocarcinoma of the colorectum: clinicopathological and genetic features. Ann Surg Oncol 2008;15:1429-1439.

7 Luey N, Toon CW, Sioson L et al. A further investigation of combined mismatch repair and BRAFV600E mutation specific immunohistochemistry as a predictor of overall survival in colorectal carcinoma. PLoS One 2014;9:e106105.

$8 \mathrm{Kim}$ SH, Shin SJ, Lee KY et al. Prognostic value of mucinous histology depends on microsatellite instability status in patients with stage III colon cancer treated with adjuvant FOLFOX chemotherapy: a retrospective cohort study. Ann Surg Oncol 2013;20:3407-3413.

9 Verhulst J, Ferdinande L, Demetter P et al. Mucinous subtype as prognostic factor in colorectal cancer: a systematic review and meta-analysis. J Clin Pathol 2012;65:381-388.

10 Inamura K, Yamauchi M, Nishihara R et al. Prognostic significance and molecular features of signet-ring cell and mucinous components in colorectal carcinoma. Ann Surg Oncol 2015;22:1226-1235.

11 Kang H, O’Connell JB, Maggard MA et al. A 10-year outcomes evaluation of mucinous and signet-ring cell carcinoma of the colon and rectum. Dis Colon Rectum 2005;48:1161-1168.

12 Xie L, Villeneuve PJ, Shaw A. Survival of patients diagnosed with either colorectal mucinous or nonmucinous adenocarcinoma: a population-based study in Canada. Int J Oncol 2009;34:1109-1115.

13 Hamilton SR, Vogelstein B, Kudo S et al. Carcinoma of the colon and rectuminHamilton SR, Aaltonen LAWorld Health Organization Classification of Tumours: Pathology and Genetics of Tumours of the Digestive System. IARC Press: Lyon, 2000;pp105-pp119.

14 Sinicrope FA, Rego RL, Halling KC et al. Prognostic impact of microsatellite instability and DNA ploidy in human colon carcinoma patients. Gastroenterology 2006;131:729-737.
15 Benatti P, Gafà R, Barana D et al. Microsatellite instability and colorectal cancer prognosis. Clin Cancer Res 2005;11:8332-8340.

16 Rosty C, Williamson EJ, Clendenning M et al. Should the grading of colorectal adenocarcinoma include microsatellite instability status? Hum Pathol 2014;45: 2077-2084.

17 Messerini L, Ciantelli M, Baglioni S et al. Prognostic significance of microsatellite instability in sporadic mucinous colorectal cancers. Hum Pathol 1999;30: $629-634$.

18 Toon CW, Walsh MD, Chou A et al. BRAFV600E immunohistochemistry facilitates universal screening of colorectal cancers for Lynch syndrome. Am J Surg Pathol 2013;37:1592-1602.

19 Yamauchi M, Morikawa T, Kuchiba et al. Assessment of colorectal cancer molecular features along bowel subsites challenges the conception of distinct dichotomy of proximal versus distal colorectum. Gut 2012;61: 847-854.

20 Colon and Rectum; in Edge SB, Byrd DR, Compton CC, Fritz AG, Greene FL, Trotti AAJCC Cancer Staging Manual. Springer: New York, 2010;pp145-pp166.

21 Hall G, Clarkson A, Shi A et al. Immunohistochemistry for PMS2 and MSH6 alone can replace a four antibody panel for mismatch repair deficiency screening in colorectal adenocarcinoma. Pathology 2010;42: 409-413.

22 Toon CW, Chou A, DeSilva K et al. BRAFV600E immunohistochemistry in conjunction with mismatch repair status predicts survival in patients with colorectal cancer. Mod Pathol 2014;27:644-650.

23 Samowitz WS, Sweeney C, Herrick J et al. Poor survival associated with the BRAF V600E mutation in microsatellite-stable colon cancers. Cancer Res 2005; 65:6063-6069.

24 Pai RK, Jayachandran P, Koong AC et al. BRAFmutated, microsatellite-stable adenocarcinoma of the proximal colon: an aggressive adenocarcinoma with poor survival, mucinous differentiation, and adverse morphologic features. Am J Surg Pathol 2012;36: 744-752.

25 Lochhead P, Kuchiba A, Imamura Y et al. Microsatellite instability and BRAF mutation testing in colorectal cancer prognostication. J Natl Cancer Inst 2013;105: 1151-1156.

26 Phipps AI, Buchanan DD, Makar KW et al. BRAF mutation status and survival after colorectal cancer diagnosis according to patient and tumor characteristics. Cancer Epidemiol Biomarkers Prev 2012;21: 1792-1798.

27 Seppala TT, Bohm JP, Friman M et al. Combination of microsatellite instability and BRAF mutation status for subtyping colorectal cancer. Br J Cancer 2015;112: 1966-1975.

28 Eklof V, Wikberg ML, Edin S et al. The prognostic role of KRAS, BRAF, PIK3CA and PTEN in colorectal cancer. Br J Cancer 2013;108:2153-2163.

29 Ito $\mathrm{M}$, Mitsuhashi K, Igarashi $\mathrm{H}$ et al. MicroRNA-31 expression in relation to BRAF mutation, CpG island methylation and colorectal continuum in serrated lesions. Int J Cancer 2014;135:2507-2515. 